\title{
Rhamnolipids-based nanostructured lipid carriers: Effect of lipid phase on physicochemical properties and stability
}

\author{
Maria A. Azevedo $^{\text {a,b }}$, Miguel A. Cerqueira ${ }^{a, *}$, Pablo Fuciños ${ }^{a}$, Bruno F.B. Silva ${ }^{a}$, \\ José A. Teixeira ${ }^{\mathrm{b}}$, Lorenzo Pastrana ${ }^{\mathrm{a}}$ \\ ${ }^{a}$ International Iberian Nanotechnology Laboratory, Av. Mestre Jose Veiga, 4715-330 Braga, Portugal \\ ${ }^{\mathrm{b}}$ Centre of Biological Engineering, University of Minho, Campus de Gualtar, 4710-057 Braga, Portugal
}

\section{A R T I C L E I N F O}

\section{Keywords:}

Nanoencapsulation

Nanotechnology

Rhamnolipids

Biosurfactants

Lipid nanosystems

\begin{abstract}
A B S T R A C T
In this work rhamnolipids were evaluated as surfactants for the production of nanostructured lipid carriers (NLCs). NLCs were produced by melt-emulsification using ultra-homogenisation followed by ultrasonication and different ratios of medium-chain-triglycerides and glycerol monostearate (lipid phase) were tested. NLCs presented sizes and polydispersity index values ranged between 97 and $120 \mathrm{~nm}$ and $0.20-0.26$, respectively. Transmission electron microscopy observations confirmed the size and the spherical morphology of the NLCs. The thermal analysis and X-ray diffraction showed that the amount of solid lipid (glycerol monostearate) influences the melting, crystallisation and enthalpy of NLCs and their degree of crystallinity. Results showed that NLCs were more stable at $4{ }^{\circ} \mathrm{C}$ and the best formulation ( $1 \%$ of water phase, $0.05 \%$ of biosurfactant and solid: liquid ratio of 10:90) was stable for 30 days. This work showed the possibility of using rhamnolipids to produce NLCs and represent an important step for the development of lipid-based nanosystems using biosurfactants.
\end{abstract}

\section{Introduction}

Lipid-based nanosystems, produced by emulsification, results of the mixing of two immiscible liquids (lipid and aqueous phases); and normally is used a surfactant to avoid the breakdown and improve the stability of the nanosystem. Lipid-based nanosystems present different features arising from the type of lipid phase used, being possible to obtain oil-in-water nanoemulsions (NEs), solid-lipid nanoparticles (SLNs) and nanostructured-lipid carriers (NLCs) (Marques et al., 2019). These nanosystems have shown to be a valuable alternative to protect the characteristics of bioactive compounds and improve their delivery and availability. They can be used to the controlled release and increased bioavailability of the bioactive compounds but also guarantee a better appearance, texture and stability of the final product as (Kuan et al., 2012). They present several advantages against other nanosystems, such as their scalability, presence of digestible lipids (that improve absorption), the possible absence of solvents and the use of food grade materials for their production (i.e. lipids, surfactants and waxes) (Tamjidi et al., 2013).

NLCs have a matrix that compromises a mixture of crystallised/solid (fat) and liquid (oil) lipids which allows having some advantages over other lipid-systems when used as carriers such as an increased dispersity and shelf-life as well as improved oral bioavailability (Babazadeh et al., 2017; Tamjidi et al., 2013). The solid lipid protects the lipophilic compounds against degradation while the liquid lipid helps to dissolve the compound and act as a physical barrier against damage factors from aqueous phases. Therefore the evaluation of solid lipid and liquid lipid ratios is of great importance for the development of nanosystems and understating of their properties.

One of the challenges when using NLCs in food applications is the use of bio-based and biodegradable food-grade materials (Babazadeh et al., 2017; Cerqueira et al., 2014). Most of the conventional synthetic surfactants are petroleum-based. Their synthesis is expensive, and usually, they are toxic to the environment, non-biodegradable and might bioaccumulate. One of the possibilities is to replace synthetic surfactants by biosurfactants (Rodrigues, 2015). Biosurfactants are naturally produced by microorganisms, being possible to use low-cost renewable feed-stocks and agro-industrial by-products and wastes as culture media. They have low toxicity, high biodegradability, high selectivity, low critical micelle concentrations and effectiveness at extreme temperatures, pHs and salinities (Banat et al., 2010; Gudiña et al., 2015; Rodrigues, 2015). Biosurfactants, similarly to synthetic surfactants, are

\footnotetext{
* Corresponding author.

E-mail address: miguel.cerqueira@inl.int (M.A. Cerqueira).
} 
amphipathic molecules that have at least a hydrophilic and a hydrophobic group and can be used to reduce the surface and interfacial tension between phases with different polarities (Gudiña et al., 2015; Henkel et al., 2017). Due to these characteristics, biosurfactants have already been proposed to the food industry as additives or emulsifiers to improve texture, organoleptic properties and creaminess of products (Banat et al., 2010). One of the best-studied biosurfactants to date are the glycolipids (e.g. rhamnolipids), composed by a sugar molecule and a lipid moiety linked with ester or amide groups. Rhamnolipids are usually produced by Pseudomonas aeruginosa (gram-negative bacteria) and their structure can have one (mono-rhamnolipids) or two (di-rhamnolipids) rhamnoses and two or more (exceptionally one) $\beta$-hydroxy fatty acids (saturated or unsaturated) with different chain lengths (C8-C24). The rhamnoses constitute the hydrophilic moiety and the fatty acid chains constitute the hydrophobic part (Gudiña et al., 2015; Henkel et al., 2017).

Some works already used rhamnolipids as surfactants for the formation and/or stabilisation of nanosystems, but most of them have been focused on nanoemulsions. Bai \& McClements (2016) used rhamnolipids to stabilise oil-in-water nanoemulsions, obtaining particles with diameters smaller than $150 \mathrm{~nm}$. Yi et al. (2019) reported the development of self-assembled oil in water $(\mathrm{O} / \mathrm{W})$ nanoemulsions using rhamnolipids as a biosurfactant with a hydrophobic photosensitiser loaded and diameters around $136.1 \mathrm{~nm}$. Müller et al. (2017) studied the potential of different rhamnolipids to form nanoparticles for dermal drug delivery and depending on the molecular structure of rhamnolipids they obtained average sizes between 5 and $100 \mathrm{~nm}$. Dai et al. (2018, 2019) and Wei et al. (2019) developed zein-nanoparticles and used rhamnolipids to stabilise and increase the stability of the nanoparticles. The use of biosurfactants for the development of NLCs is unexplored. Kanwar et al. (2019) developed NLCs (average size and polydispersity index obtained were ca. $84 \mathrm{~nm}$ and 0.46 , respectively) with sophorolipids (class of glycolipids) by the hot dispersion method to deliver the hydrophobic drug-Rifampicin. However, to the best of the authors' knowledge, there are no works using rhamnolipids for the development of NLCs. Therefore, and taking into account the potential of the rhamonolipids and the advantages of the NLCs, the main aim of this work was to evaluate the suitability of rhamnolipids produced by Pseudomonas aeruginosa to form and stabilise NLCs.

\section{Materials and methods}

\subsection{Materials}

Neobee 1053 (caprylic/capric triglyceride made using glycerin from vegetable oil sources and medium-chain fatty acids from coconut or palm kernel oils, $55 \% \mathrm{C}_{7} \mathrm{H}_{15}$ and $44 \% \mathrm{C}_{9} \mathrm{H}_{19}$ ) was kindly donated by Stepan Company (Corporate Headquarters, USA) and referred as MCT. Glycerol monostearate (GM), $>95.0 \%$ monoglyceride, was purchased from Alfa Aesar (Thermo Fisher (Kandel) GmbH, Germany). Rhamnolipids (a mixture of mono- and di- rhamnolipids with $35 \%-45 \%$ of monoand produced by Pseudomonas aeruginosa) were kindly donated by NatSurFact (NatSurFact Laboratories, USA).

\subsection{Nanotructured lipid carries (NLCs) production}

The NLCs were prepared by melt-emulsification using ultrahomogenisation followed by ultrasonication. For NLCs production, MCTs (liquid lipid, L) and GM (solid lipid, S) were used as the lipid phase (LP). The rhamnolipids (used as surfactant - Sf) were dissolved in ultrapure water and used as the aqueous phase (AP). The LP and AP were homogenised and heated at $75^{\circ} \mathrm{C}$ in different glass beakers. Then, the hot AP was added into melted LP under high shear homogenisation using an ultra-turrax (IKA Ultra-Turrax T18 digital with S18-10G dispersing element, IKA $®-W e r k e \mathrm{GmbH} \&$ Co. KG, Germany) at $14,200 \mathrm{rpm}$ for $15 \mathrm{~min}$ at $75{ }^{\circ} \mathrm{C}$. The resulting mixture was then sonicated through an ultrasonic processor (Branson Digital Sonifier Model 450, Branson Ultrasonics Corporation, USA) with $65 \%$ of amplitude for $20 \mathrm{~min}$ ( $1 \mathrm{~s}$ pulse and $1 \mathrm{~s}$ off) at $75{ }^{\circ} \mathrm{C}$. At the end the mixture was put in ice for $20 \mathrm{~min}$ for recrystallisation of the lipid phase and to form NLCs. To proceed with freeze-drying, the NLCs solutions were frozen for $24 \mathrm{~h}$ at $-20{ }^{\circ} \mathrm{C}$ and then for more $24 \mathrm{~h}$ at $-80^{\circ} \mathrm{C}$. The freeze-drying was carried out using a Freeze Dryer Lyoquest $-55^{\circ} \mathrm{C}$ Plus Eco (Telstar Technologies S.L.U, Spain). During freeze-drying, the temperature and the vacuum were maintained at $-50{ }^{\circ} \mathrm{C}$ and $0.100 \mathrm{mbar}$, respectively. The dried NLCs obtained were stored in a desiccator with silica at room temperature until further use. Fresh produced NLCs were evaluated by DLS, turbidity, SAXS and TEM and dehydrated NLCs were evaluated by XRD and DSC.

The optimisation of the formulations was based on a Face-Centered Central Composite design (Box et al., 2005). The ratio of aqueous:lipid phases, the ratio solid:liquid lipids, and the concentration of rhamnolipids were used as the independent variables and the NLC's size (by intensity) and PDI were used as the response variables (Supplementary Material). The minimum predicted size and PDI in this region occurred at $\mathrm{AP}=99 \%, \mathrm{~S}=20 \%$ and $\mathrm{Sf}=0.05 \%$, therefore, in an attempt to reduce the size and obtain a monodisperse distribution (PDI $<0.2$ ), two additional conditions were assayed in which ratio of the aqueous:lipid phase and the concentration of biosurfactant were maintained as in the predicted minimum ( $\mathrm{AP}=99 \%, \mathrm{Sf}=0.05 \%$ ) and the effect of the solid: liquid lipid ratio (S) was brought down to $15 \%$ and $10 \%$. Therefore, were selected three formulations: NLCs 1, NLCs 2 and NLCs 3 with S of $20 \%, 15 \%$ and $10 \%$, respectively, being the percentage of AP (99\%) and Sf $(0.05 \%)$ the same for all the formulations.

\subsection{Physicochemical characterisation of NLCs}

\subsubsection{Nanoparticle size, polydispersity index and zeta potential}

Size, polydispersity index (PDI) and zeta potential $(\zeta)$ were measured with a Dynamic Light Scattering (DLS) apparatus (SZ-100Z, Horiba Instruments Inc., USA). For size and PDI determination, polystyrene cuvettes were used. For the zeta potential an electrode cell of carbon with $6 \mathrm{~mm}$ was used. All measurements were performed using fresh samples with a dilution of $10 \mathrm{x}$ at $25{ }^{\circ} \mathrm{C}$ and each measurement of size (by intensity) and PDI were measured with a detection angle of $90^{\circ}$ being the zeta potential performed with a detection angle of $173^{\circ}$. The refractive index used was 1.6. For all measurements at least five replicates were performed.

\subsubsection{Turbidity measurements}

To evaluate the stability of NLCs the turbidity was measured through the absorbance at $600 \mathrm{~nm}$ using NanoDrop spectrophotometer (NanoDrop 3300, Thermo Fisher Scientific, USA). The measurements were performed at $25{ }^{\circ} \mathrm{C}$ using a quartz cuvette with a path length of $10 \mathrm{~mm}$ and distilled water as a blank. Before measurements, the fresh NLC samples were diluted 10x. At least five measurements were performed per sample.

\subsubsection{Small angle $X$-ray scattering (SAXS)}

Structural and crystallinity changes over time were characterised by small angle X-ray scattering (SAXS). In SAXS a range of scattering vectors $q$ is scanned where electron density periodicities with spacing $d$ result in scattering peaks at values of $q=2 \pi / d$. Besides the determination of such structures' periodic repeat distances $d$, an approximate idea of their domain size can be obtained through the width of the scattering peak. The half-width at half maximum $(\delta)$ of the diffraction peak is determined by fitting the peak with a Lorentzian function (Equation (1)) (Bouxsein et al., 2011).

$$
I(q)=\frac{A}{\delta^{2}+(q-q 0)^{2}}+C_{0}
$$


where, $q$ is the scattering vector, $I(q)$ is the scattering intensity as a function of $q, q_{0}$ is the $q$ value at the maximum peak height, $A$ is a proportionality constant related to the peak height, and $C_{0}$ is a constant related with background scattering. With $\delta$ determined by fitting the scattering peaks with Equation (1), the domain size $d_{s}$ is then estimated according to Equation (2).

$d_{s}=\frac{\pi}{\delta}$

The domain size $d_{s}$ provides a length over which the structure is coherent. For 1D layered structures (e.g. lamellar and coagel phases), dividing $d_{s}$ by the structure repeat distance $d$ provides the number of coherent lamellar layers $n_{l}$ (Silva et al., 2014).

NLCs in solution were used for the measurements, being the samples prepared in sealed capillaries (Hilgenberg, Germany) without any dilution. The samples were measured with a SAXSess $\mathrm{mc}^{2}$ Kratky camera (Anton Paar GmbH, Austria) using Cu-Ko radiation $(\lambda=1.54056 \AA)$ and operating at $40 \mathrm{kV}$ and $50 \mathrm{~mA}$. An image plate system covering the $q$ range from 0.23 to $26 \mathrm{~nm}^{-1}$ was used. The exposure time for all samples was $60 \mathrm{~min}$ being the temperature of $25{ }^{\circ} \mathrm{C}$. The measurements were performed in line-collimation mode to maximise scattering intensity. The 2D scattering images were converted to $1 \mathrm{D}$ radial intensity profiles using the SAXS-quant software (Anton Paar GmbH, Austria).

\subsubsection{Transmission electron microscopy (TEM)}

The morphology of the NLCs was evaluated by transmission electron microscopy (TEM) (JEOL JEM 2100 - HT - 80-200 kV LaB6 gun, JEOL Ltd, Japan). The images were digitally recorded using an UltraScan ${ }^{\circledR}$ 4000 CCD camera (Oneview, Gatan, USA). The aliquots were deposited onto grids coated with an ultrathin carbon film (400 mesh, approx. grid hole size of $42 \mu \mathrm{m}$, PELCO ${ }^{\circledR}$, TED PELLA INC., USA) and UranyLess EM Stain (Electron Microscopy Sciences (EMS), USA) was used as the contrast agent. The samples dried at room temperature and $24 \mathrm{~h}$ later micrographs of the samples were taken.

\subsubsection{Stability during storage}

In order to evaluate the stability of NLCs in solution, fresh samples were stored at different temperatures $\left(4^{\circ} \mathrm{C}, 25^{\circ} \mathrm{C}\right.$ and $\left.37^{\circ} \mathrm{C}\right)$. For this, the size, PDI, zeta potential and turbidity were measured, while the morphology and structural changes were determined by TEM and SAXS, respectively.

\subsubsection{Differential scanning calorimetry (DSC)}

The crystalline state of the freeze-dried NLCs was evaluated by differential scanning calorimetry (DSC) using a DSC calorimeter (Perkin Elmer DSC 6000, Perkin Elmer, USA). The samples were weighed (4-5 $\mathrm{mg}$ ) and loaded onto sample aluminium pans of the DSC instrument. An empty pan was used as a reference. Heating and cooling steps were performed as follow: a) $1^{\text {st }}$ cooling step the samples started at $25^{\circ} \mathrm{C}$ and cooled to $-45{ }^{\circ} \mathrm{C}$, b) $1^{\text {st }}$ heating step the samples were heated from $-45{ }^{\circ} \mathrm{C}$ to $95{ }^{\circ} \mathrm{C}$, and c) $2^{\text {nd }}$ cooling step the samples were cooled from $95{ }^{\circ} \mathrm{C}$ to $25^{\circ} \mathrm{C}$. All steps were performed at $10^{\circ} \mathrm{C} /$ minute under a nitrogen atmosphere with a flow rate of $20 \mathrm{~mL} / \mathrm{min}$. Before DSC measurements the equipment was calibrated with indium and zinc standards of known melting temperature and enthalpy $\left(156.6^{\circ} \mathrm{C}, 28.45 \mathrm{~J} / \mathrm{g}\right.$ and $419.47{ }^{\circ} \mathrm{C}, 108 \mathrm{~J} / \mathrm{g}$, respectively). The final thermograms are derived from baseline subtraction (performed with an empty furnace) in order to obtain absolute values of the sample heat flow. The obtained thermograms were analysed using the Pyris software (version 13) to determine the transition temperatures and respective enthalpies. Each sample analysis was performed at least in duplicate.

\subsubsection{X-ray diffraction (XRD)}

The crystalline profiles of freeze-dried NLCs were studied by X-ray diffraction (XRD), using a Malvern Panalytical X Pert PRO MRD diffractometer using $\mathrm{Cu}-\mathrm{K} \alpha$ radiation $(\lambda=1.54056 \AA)$ and operating at
$45 \mathrm{kV}$ and $40 \mathrm{~mA}$. The patterns were collected at room temperature in a $2 \theta$ range from $5.0^{\circ}$ to $50^{\circ}$ being the step size of $0.02^{\circ} / \mathrm{s}$. The fine calibration offset for $2 \theta=-0.0372^{\circ}$. Information was collected during 174 $\mathrm{s}$ and the PANanalytical X'Pert HighScore Plus software was used to gather data and the analysis of peak diffractions.

\subsection{NLCs rehydration}

Dried NLCs obtained by freeze-drying were rehydrated in distilled water. First, $25 \mathrm{~mL}$ of distilled water was added to dried NLCs and then the samples were put in ultrasonic bath (Ultasonic cleaner USC $500 \mathrm{TH}$, $45 \mathrm{kHz}$ and $200 \mathrm{~W}$, VWR International Ltd, UK) during $45 \mathrm{~min}$. After that, the samples were stirred during $5 \mathrm{~h}$ at $400 \mathrm{rpm}$ and then were put back more $45 \mathrm{~min}$ on ultrasonic bath. At the end and before to measure the sizes and PDI using DLS, the samples were stirring more $5 \mathrm{~min}$ at 400 $\mathrm{rpm}$. The morphology of rehydrated NLCs was also evaluated by TEM.

\subsection{Statistical analysis}

Statistical analyses were performed using Microsoft Excel 2003 and GraphPad Prism (Version 5.00 (Trial), edition 2007, GraphPad Software, Inc., La Jolla, CA, USA). All the experiments subjected to statistical analyses were carried out at least in duplicate. The statistical analyses were performed using analysis of variance (ANOVA) followed by Tukey's multiple-comparison test $(\alpha=0.05)$ to assess the statistical significance of differences between groups of data. Differences in experimental results were considered to be statistically significant at 95\% confidence level $(p<0.05)$.

\section{Results and discussion}

\subsection{Particle size, polydispersity index (PDI), zeta potential and turbidity}

Based on the optimisation presented in the Supplementary Material, three formulations were selected: NLCs 1 AP:LP (\%) = 99:1, S:L (\%) = 20:80, Sf $=0.05 \%$; NLCs 2 AP:LP (\%) = 99:1, S:L (\%) =15:85, Sf $=$ 0.05\%; and NLCs 3 AP:LP (\%) = 99:1, S:L (\%) =10:90, Sf $=0.05 \%$.

It is observed that the sizes of the particles increase significantly ( $p<$ 0.05 ) when the amount of solid lipid decreases and the liquid lipid increases, reaching size values of $97.43 \mathrm{~nm}, 111.13 \mathrm{~nm}$ and $118.15 \mathrm{~nm}$ for NLCs 1, 2 and 3, respectively (Table S5 in Supplementary Material). The increase of NLCs' size can be explained by the higher amount of liquid lipid that leads to a more disordered and less dense NLC structure ( $\mathrm{Hu}$ et al., 2006). Another possible reason is the increase of interfacial tension or swelling of the core of the NLCs as a result of the increase of liquid lipid concentration (Soleimanian et al., 2018). However, this difference is not observed between the NLCs with $15 \%$ and $10 \%$ of solid lipid. Regarding the PDI, NLCs presented values ranged between 0.22 and 0.25 , which are not statistically $(p>0.05)$ different between each other (Table S5 in Supplementary Material). PDI values bellow 0.25 mean that NLCs have a fairly narrow size distribution which can favour their long-term stability (Tamjidi et al., 2013).

Results showed that the turbidity values increase for higher sizes of NLCs (Table S5 in Supplementary Material). These results are in agreement with the work of Uluata et al. (2016) that observed that emulsions with diameters around $45 \mathrm{~nm}$ had smaller turbidity values than emulsions with diameters around $216 \mathrm{~nm}$. This increase is related with the scatter light that is higher for bigger particles than smaller ones. These values will be important to define future applications of NLCs since will change optical properties of the final products.

NLCs showed a high negative zeta potential (Table S5 in Supplementary Material) with values ranging from 76.64 and $88.04 \mathrm{mV}$. The negative charge can be attributed to rhamnolipids. They have carboxylic acid groups with $\mathrm{pK}_{\mathrm{a}}$ values around $4-5$, in this way when they are in a water based systems with $\mathrm{pH}$ above 5 the carboxylic acid groups are charged (-COO$\left.{ }^{-}\right)$making the rhamnolipids anionic (Bai \& McClements, 

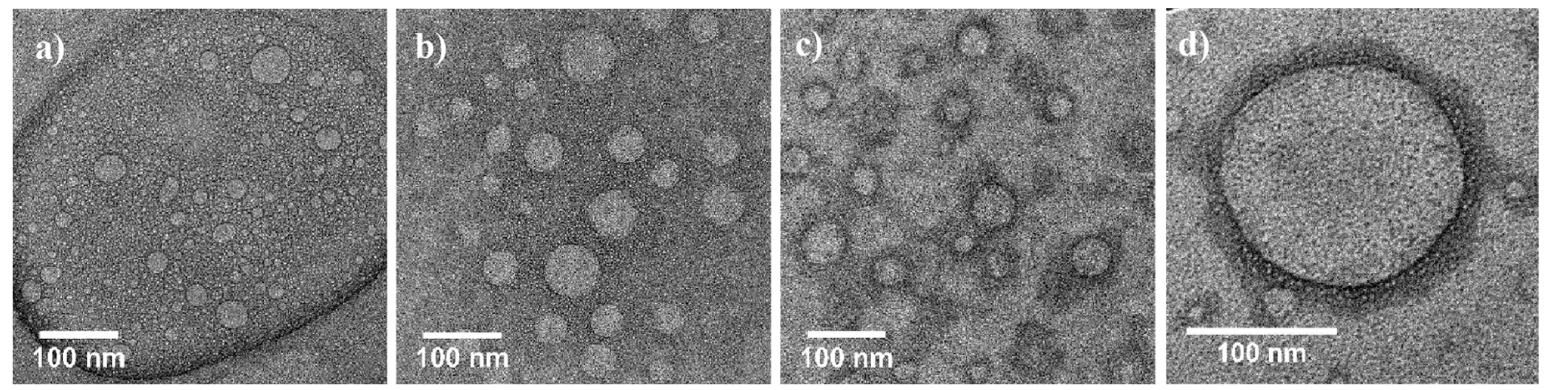

Fig. 1. Transmission electron microscopy (TEM) images of NLCs: a) NLCs 1; b) NLCs 2; c) and d) NLCs 3. Scale bar: 100 nm and magnification of $100000 x$.

2016). Through the zeta potential values is possible to assess the colloidal stability of nanoparticles. Usually, nanoparticles with zeta potentials above $+30 \mathrm{mV}$ or below $-30 \mathrm{mV}$ are considered stable (Azevedo et al., 2014).

\subsection{Transmission electron microscopy}

Fig. 1 shows that the morphology of NLCs with $10 \%, 15 \%$ and $20 \%$ of solid lipid is spheric and confirm the sizes obtained by DLS. However, in some cases, such NLC 3, the sizes observed are smaller than the sizes obtained by DLS. This fact can be explained by the PDI of NLCs and by the drying step needed during sample preparation which can results in smaller particle sizes.

\subsection{Stability over time}

The effect of different solid:liquid lipid ratios in NLCs' stability was evaluated over time at different temperatures $\left(4^{\circ} \mathrm{C}, 25^{\circ} \mathrm{C}\right.$ and $\left.37^{\circ} \mathrm{C}\right)$. Results showed that the NLCs stability depends not only on the liquid: solid lipid ratio, but also on the storage temperature (Fig. S1 and Table S6 in Supplementary Material). In general, all the NLCs maintained their size, PDI, zeta potential and turbidity values for a longer time when the storage temperature was $4{ }^{\circ} \mathrm{C}$; however when the NLCs are stored at high temperatures, the kinetic energy of the system, that is higher, accelerate the collision of the particles, resulting in aggregation and destabilisation of the NLCs (Hu et al., 2006). In general, this destabilisation was more evident in the PDI values than on the sizes, which could hint at an Ostwald ripening mechanism of destabilisation, in which a small (but finite) solubility of GM or MCTs in water could induce migration from smaller NLCs to larger NLCs, accentuating the increase of PDI over time (Egelhaaf et al., 1999). Observing the sizes of NLCs over time it is possible to see that the NLCs maintained their sizes during the storage $(p>0.05)$. However, the high PDI values observed at the end of the evaluation tests and the increase of turbidity over time ( $p$ $<0.05$ ) show an instability/heterogeneity of the samples during storage. Besides that, a decrease of zeta potential over time $(p<0.05)$ was also observed for all storage temperatures and all the formulations (with exception of the NLCs 3 at $37^{\circ} \mathrm{C}$ ). This decrease can be explained by the increase if the kinetic energy which can lead to changes in the crystalline structure of the lipid (Hu et al., 2006) and therefore results in the decrease of zeta potential.

Regarding the effect of different solid:liquid lipid ratios in the NLCs' stability, a decrease in the stability in the following order NLCs $3>$ NLCs $2>$ NLCs 1 was observed. At the end of the stability test at $25{ }^{\circ} \mathrm{C}$ (29 days) a phase separation was observed. This was more evident for the NLCs 1 as can be seen in Fig. S2. The same happened for the other tested temperatures. These observations show that the NLCs are more stable for lower concentrations of GM.

TEM and SAXS were also used to evaluate the NLCs morphology and structural changes of the NLCs, during storage. All the formulations present relatively featureless SAXS profiles for early times of storage (Fig. S3 in Supplementary Material). For all formulations, a scattering
Table 1

Crystallinity properties ( $q_{0}-$ momentum transfer that corresponds to maximum intensity; $d$ - distance between the layers ( $d$-spacing); $d_{s}$ - domain size) of NLCs 1,2 and 3 during storage time at different temperatures $\left(4,25\right.$ and $\left.37^{\circ} \mathrm{C}\right)$.

\begin{tabular}{clllll}
\hline & & $\begin{array}{l}\text { Time } \\
\text { (Days) }\end{array}$ & $\begin{array}{l}q_{0} \\
\left(\mathrm{~nm}^{-1}\right)\end{array}$ & $\begin{array}{l}d \\
(\mathrm{~nm})\end{array}$ & $\begin{array}{l}d_{\mathrm{s}} \\
(\mathrm{nm})\end{array}$ \\
\hline $4^{\circ} \mathbf{C}$ & 20 & 1.32 & 4.76 & 59.84 \\
\cline { 2 - 6 } & NLCs 1 & 31 & 1.34 & 4.69 & 41.89 \\
& NLCs 2 & 45 & 1.32 & 4.78 & 54.17 \\
\hline $\mathbf{2 5}{ }^{\circ} \mathbf{C}$ & NLCs 3 & 45 & 1.31 & 4.80 & 44.88 \\
& & 60 & 1.32 & 4.75 & 69.81 \\
\cline { 2 - 6 } & NLCs 1 & 11 & 1.30 & 4.83 & 36.96 \\
& & 20 & 1.29 & 4.87 & 39.27 \\
\cline { 2 - 6 } & NLCs 2 & 20 & 1.31 & 4.78 & 66.84 \\
\cline { 2 - 6 } & NLCs 3 & 20 & 1.32 & 4.76 & 89.76 \\
& & 29 & 1.32 & 4.78 & 89.76 \\
\hline \multirow{3}{*}{${ }^{\circ} \mathbf{C}$} & NLCs 1 & 6 & 1.31 & 4.80 & 37.85 \\
& & 10 & 1.30 & 4.83 & 52.36 \\
\cline { 2 - 6 } & NLCs 2 & 10 & 1.31 & 4.80 & 52.36 \\
& & 17 & 1.29 & 4.86 & 52.36 \\
\hline
\end{tabular}

peak at $\mathrm{q} \sim 1.3 \mathrm{~nm}^{-1}$ emerges with time, signalling structural changes within the NLCs during storage. The emergence of this peak is linked with the observed changes in size, zeta potential, PDI and turbidity, as well as with the visual observations of macroscopic phase separation (Fig. S2). Hence, this peak is intrinsically linked with the destabilisation process. This peak corresponds to real space periodicities of $d=4.7$ to $4.9 \mathrm{~nm}$. The domain sizes calculated with Equation (1) have values between 37 and $90 \mathrm{~nm}$. These domains can be seen as nano-sized crystallites, increasing in size with time (Table 1). However, even at the last measurements their sizes were smaller than the estimated NLCs size.

It is noted that the periodicities measured in the NLCs $(d=4.7-4.9$ $\mathrm{nm}$ ) are very close to the periodicities observed for the $\beta$ (or coagel) phase of the GM below $57{ }^{\circ} \mathrm{C}$ (Wang \& Marangoni, 2014), which corresponds to a lamellar arrangement of the GM bilayers with the chains slightly tilted $(d=4.9 \mathrm{~nm})$. Hence the emerging structure in the present case could be a coagel phase consisting mostly of GM that segregates with time. Because in the present case, the second reflection expected for the coagel phase is not observed, this phase assignment is not certain.

Given the close similarity of the $d$ spacing with the one expected for the $\beta$ phase of GM, it can be said that with time the GM starts slowly nucleating into the $\beta$ phase, coexisting with the liquid lipid phase (a mixture of MCT and GM). With time the crystallites grow significantly in size resulting in a peak observable with SAXS. If the structure is indeed coagel, the number of layers $\left(n_{l}\right)$ determined by dividing $d_{s}$ by $d$ would be ca. 8-19 layers (Equation (1)). It is not clear if this slow nucleation is a cause of instability or a consequence from another process. The instability can indeed result from other mechanisms, such as Ostwald 

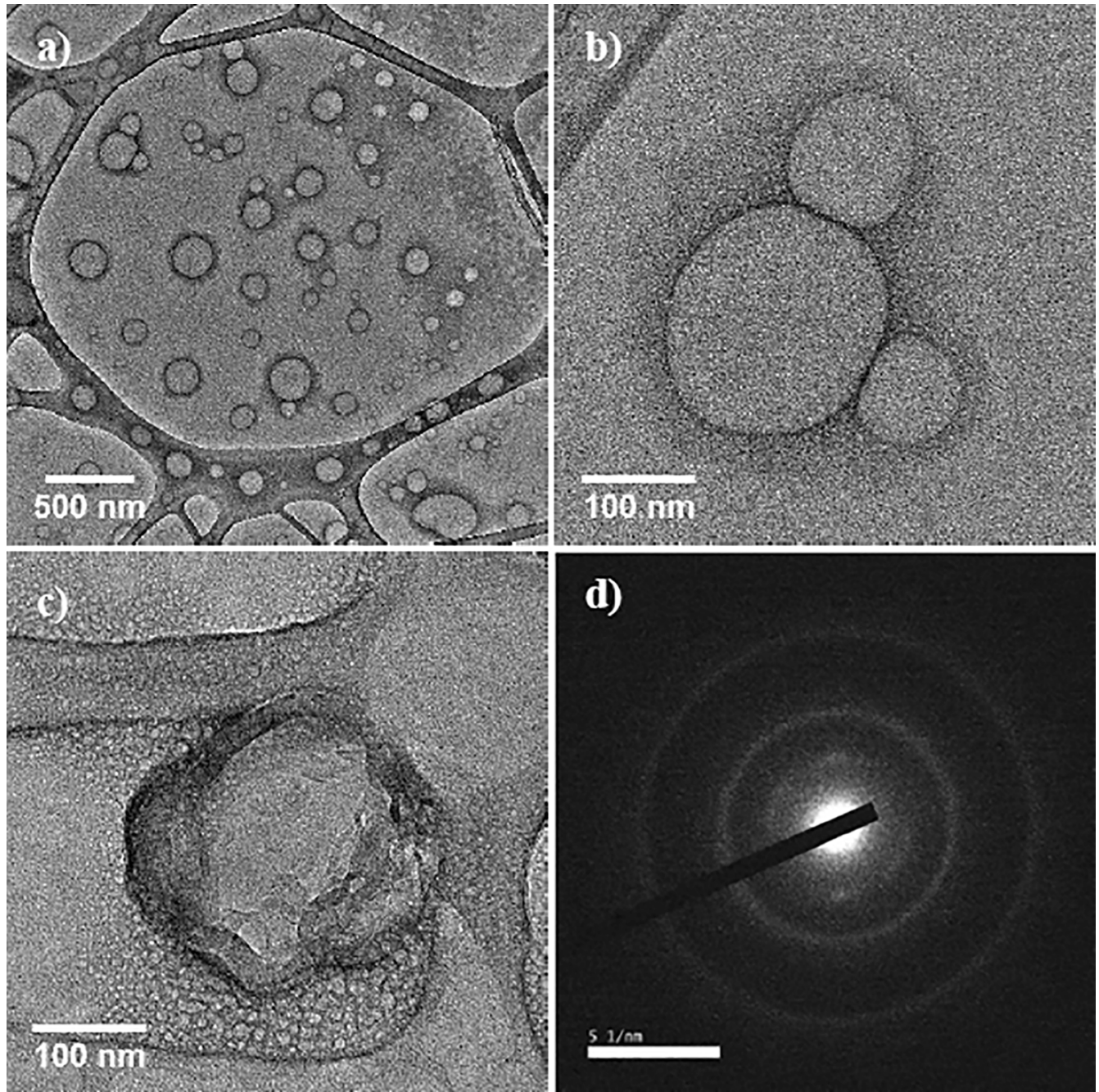

Fig. 2. Transmission electron microscopy (TEM) images of NLC 3 after 31 days of storage at $4{ }^{\circ} \mathrm{C}(\mathrm{a}, \mathrm{b}$ and c) and the Fig. $2 \mathrm{~d}$ is the electron diffraction of the structure (NLCs 3). Scale bar: a) $500 \mathrm{~nm}, 150000 x$; b) $100 \mathrm{~nm}, 100000 x$; c) $100 \mathrm{~nm}, 80000 x$.
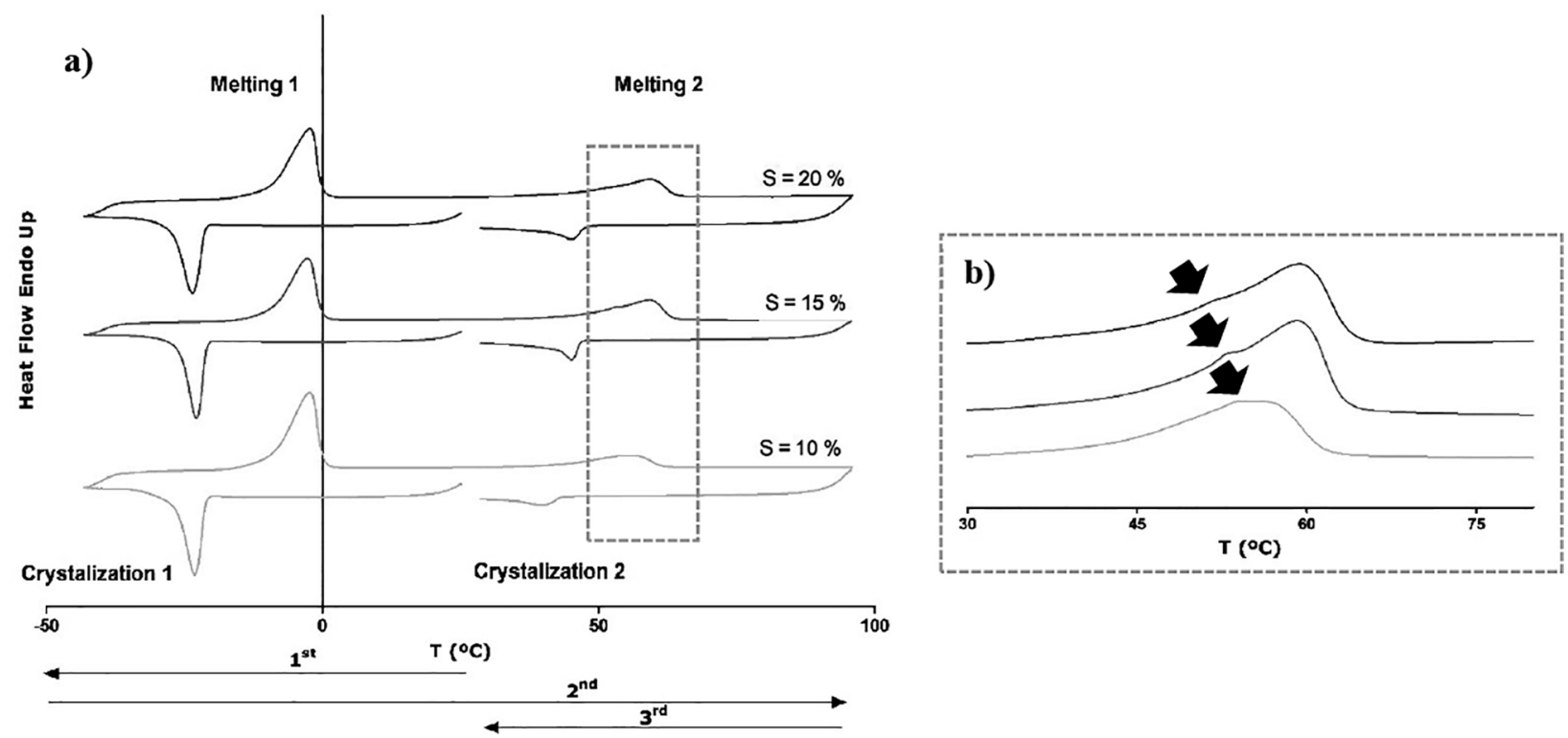

Fig. 3. Differential scanning calorimetry (DSC) thermograms of NLCs using different percentage of solid lipid ( $\mathrm{S}=20 \%, \mathrm{~S}=15 \%$ and $\mathrm{S}=10 \%$ ). a) NLCs 1 ( $\mathrm{S}=20 \%$ ), NLCs $2(S=15 \%)$ and NLCs $3(S=10 \%)$ throughout the heating and cooling ramps and b) endothermic peak observed from 30 to $80^{\circ} \mathrm{C}$. 


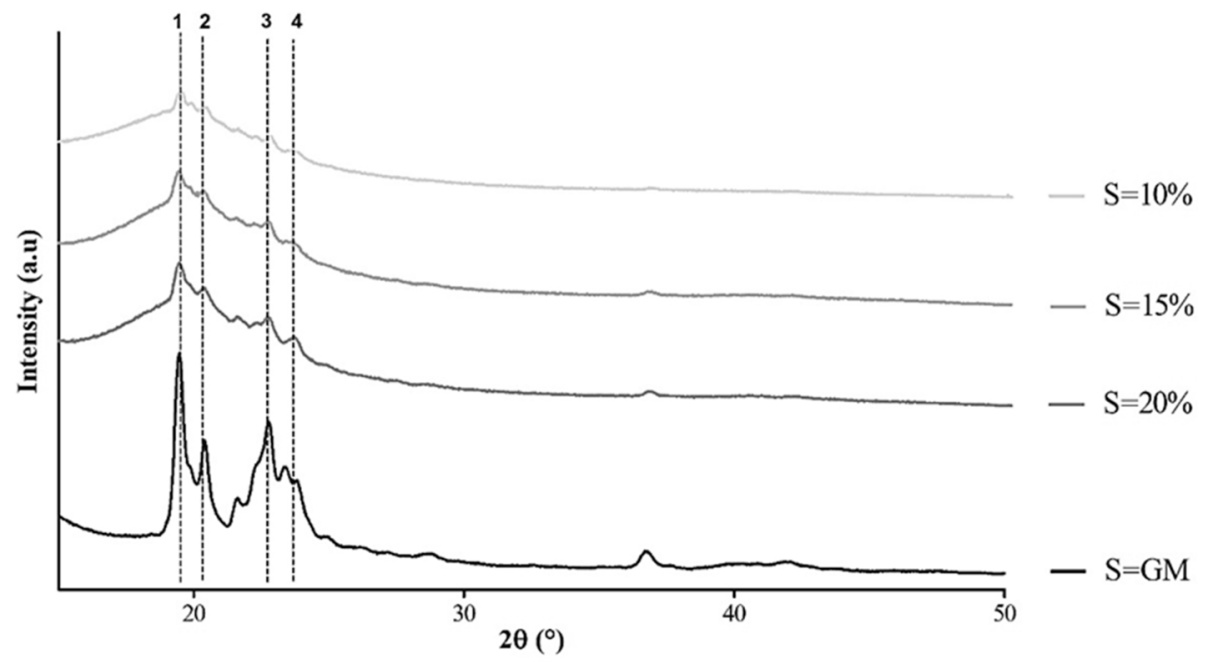

Fig. 4. X-ray profiles of glycerol monostearate (GM) and freeze-dried NLCs 1 (S = 20\%), NLCs 2 (S = 15\%) and NLCs 3 (S = 10\%).

ripening, in which e.g. a small (but finite) solubility of GM in water would result in migration between NLCs, resulting in the growth of some of the particles and shrinkage of others.

The morphology of NLCs, during the storage time, was evaluated by TEM. TEM images (Fig. 2) showed that some effects like coalescence and aggregation occurred over time and it was possible to see an increase of heterogeneity of the samples, which is in agreement with the PDI values obtained. Some samples also showed residues and using the electron diffraction was possible to see that these residues were polycrystalline materials what can be related to GM loss from NLCs structure.

\subsection{Thermal analysis}

The solid (GM) and liquid (MCT) lipids present different melting/ crystallisation temperatures and due to those differences, they can influence the NLCs thermal behaviour. Fig. 3a shows the DSC curves of NLCs produced (Fig. S4 presents the DSC curves of the raw materials used). All NLCs present four peaks: two peaks that correspond to melting temperatures and two peaks that correspond to crystallisation temperatures. The two melting/crystallisation peaks result from the two materials used to develop NLCs. The MCT is responsible for the first exothermic and endothermic events (crystallisation 1 and melting 1) and the GM originates the second endothermic and exothermic events (melting 2 and crystallisation 2). The crystallisation temperatures are always significantly lower than the corresponding melting temperatures (i.e. there is an undercooling effect). This is explained by the formation of ordered phases on cooling that requires the emergence of nucleation points, whereas on heating the order-disorder transitions do not require nucleation. Hence, when comparing the transition temperatures, it will be used the two melting temperatures $\left(\mathrm{T}_{\mathrm{m} 1}\right.$ and $\left.\mathrm{T}_{\mathrm{m} 2}\right)$. When comparing the melting temperatures and enthalpies of the NLCs with those of the neat MCT and GM materials it is observed a shift in the temperature peaks. Regarding the temperatures of melting $2\left(\mathrm{~T}_{\mathrm{m} 2}\right)$, where the GM is the main responsible, it is possible to see in Fig. 3a (Table S7 in Supplementary Material) that the melting temperatures of NLCs were lower than those observed in pure GM and the decrease of temperatures follow the reduction of GM concentration. The same behaviour was observed with the temperatures of crystallisation $2\left(\mathrm{~T}_{\mathrm{c} 2}\right)$ and their enthalpy values. Relatively to the first exothermic and endothermic events $\left(\mathrm{T}_{\mathrm{m} 1}\right.$ and $\mathrm{T}_{\mathrm{c} 1}$ ) it was observed a decrease for lower GM concentrations and consequence higher MCT concentrations.

Fasolin et al. (2018) studied the thermodynamic properties of selfsustainable structured oils using different gelators with medium-chain triglycerides or high oleic sunflower (MCT and LCT, respectively).
They tested a blend of 15 and $20 \%$ of GM with MCT and observed that the gelator concentration exerted influence on the thermodynamic parameters. The blend with $20 \%$ of GM presented melting and crystallisation temperatures and enthalpies higher than the blend with $15 \%$. They observed that both blends had thermodynamic parameters lower than the pure gelator. In present work, the thermodynamic parameters were also influenced by the concentration of GM and the same behaviour was observed. Thus, the difference between pure GM and the blend of GM with MCT or the NLCs can be explained by an eutectic effect that is characteristic in this type of materials. Eutectic effects tend to occur when the constituents of the systems present incompatibilities like molecular volumes, shapes and/or polymorphs. The consequence of that is a decrease in the melting and crystallisation temperatures when compared with pure components. This effect can be beneficial for NLCs because the less compact crystalline nanostructures may favour the encapsulation of bioactive compounds (Himawan et al., 2006; Santos et al., 2019).

The increase of the MCT amount can also explain the decrease of melting and crystallisation temperatures $\left(\mathrm{T}_{\mathrm{m} 2}\right.$ and $\mathrm{T}_{\mathrm{c} 2}$, respectively) of NLCs, which is justified by the formation of less ordered crystals. The addition of oil to a lipid matrix origin defects in the crystalline lattice of the solid lipid and as consequence NLCs with less ordered crystalline arrangement are formed. As mentioned before, the enthalpies follow this tendency which is also explained by the incorporation of the liquid lipid into the solid matrix, which leads to a reduction of the crystallinity of the lipid matrix (Nahr, Ghanbarzadeh, Hamishehkar, \& Kafil, 2018; Salminen et al., 2013). Looking in detail to the second endothermic peak, it is possible to see a shoulder that is more pronounced when the concentration of MCT is higher (black arrows in Fig. 3b). This fact can be related to a polymorphic transition of NLCs that is related with a less ordered crystalline structure. This structure can be formed due to the chemical heterogeneity and high level of liquid lipid component in NLCs. Normally, the polymorphism happens when higher concentrations of minor components are used in NLCs' development (Nahr et al., 2018). This behaviour is in agreement with other works (Castelli et al., 2005; Jenning et al., 2000) where glyceride was used to produce nanostructures, with solid and liquid lipids. They observed a decrease of the melting point of the solid lipid for higher concentrations of the liquid lipid, which indicate the association of the two lipids within the single nanoparticles after crystallisation. Even though the interaction of the two matrix components cannot be straightforwardly obtained from the DSC data for this type of mixture, in this work it is also possible to conclude that occurs an association between the GM and the MCT and therefore a structure with less ordered crystals. 


\subsection{X-ray diffraction}

Fig. 4a shows the XRD profiles of lyophilised NLCs with different percentage of solid lipid (GM) where it is possible to see that all NLCs and the GM exhibited the same patterns. Therefore, it is possible to say that GM is the main responsible for the crystallinity of NLCs. Triacylglycerides like GM, upon cooling, can crystallise into three predominant forms (polymorphism), including the unstable $\alpha$-form, the metastable sub- $\alpha$-form (also called $\beta$ '-form), and the most stable $\beta$-form. Besides the stability, the different forms have different structures; for example, the $\alpha$-form has a disordered structure while the $\beta$-form structure is more densely packed. This fact results in polymorphs with different properties like crystallisation rate, crystal size and crystallinity (Teeranachaideekul et al., 2007). Taking into account the polymorphism of triacylglycerides, their XRD patterns could be described as follows: a strong short spacing at $4.2 \AA$ for the $\alpha$-form (hexagonal subcell); a strong spacing near of $4.3 \AA$ and several spacings from 3.9 to $3.7 \AA$ to $\beta$ '-form (orthothombic perpendicular); or a strong short spacing at $4.6 \AA$ combined with several spacings from 3.9 to $3.6 \AA$ to $\beta$-form (triclinic parallel) (Weiss et al., 2008; Zheng et al., 2013). Fig. 4 shows that GM and all NLCs exhibited peaks at scattering angles (20) of ca. 19.5, 20.4, 22.8 and $23.4^{\circ}$, with corresponding interplanar distances (d-spacing) of ca. 4.6, 4.3, 3.9 and $3.8 \AA$, respectively. These values indicate the presence of $\beta$ '- and $\beta$-forms. This fact shows that the NLCs maintained the polymorphism of GM and the stable crystal lattice. The decrease of peak intensities for lower GM concentrations is a result of smaller amounts of GM crystalline phases relatively to amorphous regions composed by the MCT. The full width at half maximum (FWHM) presented in Table S8 in Supplementary Material also indicated that, although the crystal remains the same for GM and all NLCs, the size is different. The FWHM is related to the width of the peak being possible to calculate the crystallite size using this parameter and the Scherrer equation (Nahr et al., 2018):

$D=\frac{k \lambda}{2 \delta \cos \theta}$

where $\mathrm{D}$ refers to the crystal dimension, $k$ to medium factor considered as $0.9, \lambda$ to the wavelength of X-ray and $2 \delta$ to FWHM. The equation shows that the crystal dimension varies inversely with FWHM. In this case, the FWHM decreased for NLCs with lower concentrations of GM (Table $\mathbf{8 8}$ in Supplementary Material) which is explained by the increase of the crystal size. NLCs crystallinity was mainly influenced by the presence of GM in the structure; it is observed that lower concentrations of GM (and consequently high MCT concentration) in lipid phase result in NLCs with a less ordered crystalline structure and bigger crystals. The less crystalline state of NLCs can contribute to increase the stability and the loading of bioactive compounds. Besides that, this fact can also minimise the release of the compounds during storage (Nahr et al., 2018). Considering the structure of the lipid matrix, the NLCs can be denominated like imperfect (lipid crystallisation is less ordered), amorphous (matrix is solid but nor crystalline) or multiple types (solid lipid matrix contains small oil compartments) (Beloqui et al., 2017). This way, the NLCs produced in the present work can be considered as imperfect type.

\subsection{Rehydration of NLCs}

The presence of water in nanostructures like NLCs can accelerate the degradation of the lipids that are used to form this type of nanoparticles. This way, the use of freeze-drying can be one way to ensure the stability, storage, handling and the use of a solid dosage form of NLCs (Varshosaz, Eskandari, \& Tabbakhian, 2012). However, it is also important to confirm that after freeze-drying and rehydration the NLCs maintain their properties. Therefore, NLCs (1, 2 and 3 ) were dried using freeze-drying and then rehydrated in water, and their size, PDI and morphology analysed in order to know the effect of freeze-drying and rehydration.
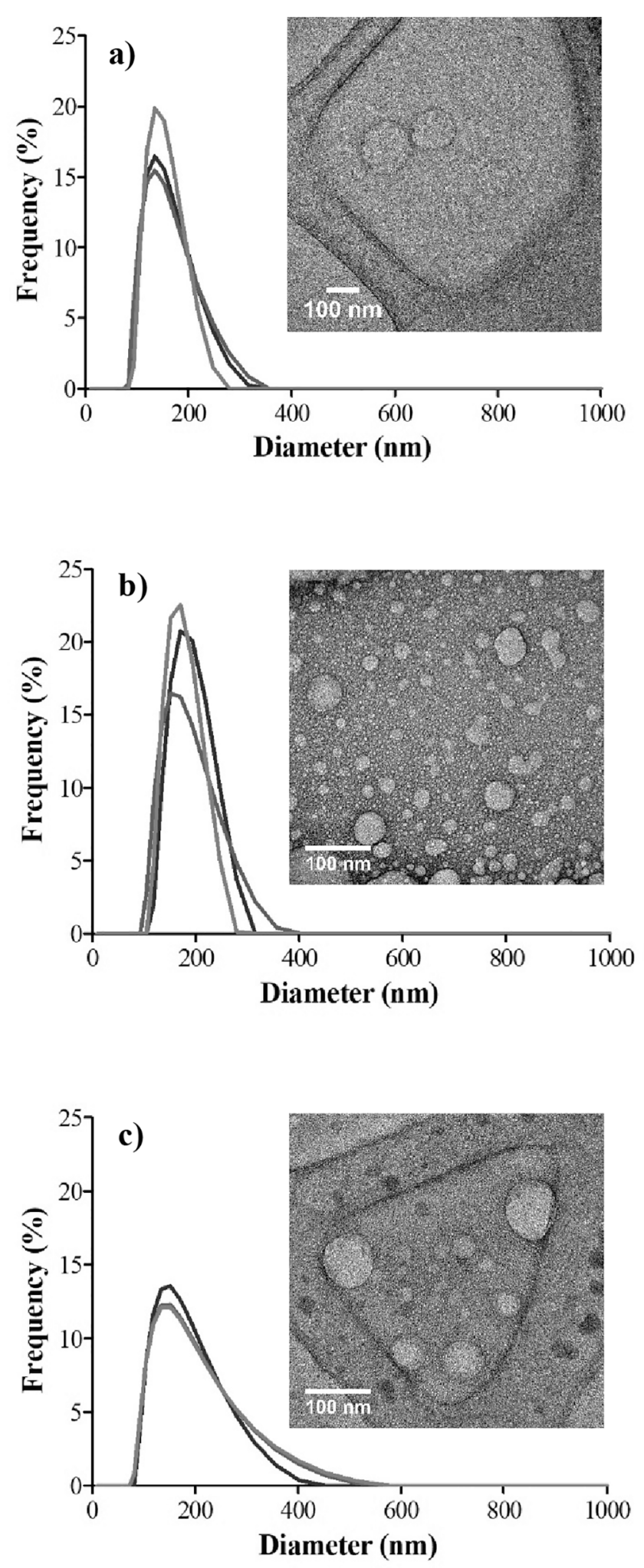

Fig. 5. Size distribution determined by DLS for each one of the rehydrated NLCs (1, 2 and 3) dried by freeze-drying and their respective TEM images: a) NLCs 1; b) NLCs 2; c) NLCs 3. Scale bar: a) $100 \mathrm{~nm}, 50000 \mathrm{x}$; b) $100 \mathrm{~nm}$, 100000x; c) $100 \mathrm{~nm}, 100000 \mathrm{x}$.

Fig. 5 shows the size distribution determined by DLS and the corresponding TEM images for each one of the rehydrated NLCs. The size distribution and the TEM images show a small increase (around $50 \mathrm{~nm}$ ) in the size distribution $(p<0.05)$ when compared with the values 
obtained for NLCs before the freeze-drying (Fig. S5). However, the PDI values before and after the freeze-drying are between 0.2 and 0.3 and do not show statistical difference $(p>0.05)$. This means that the NLCs maintain the fairly narrow size distribution after the rehydration and freeze-dried can be used for the dehydration of NLCs.

\section{Conclusions}

In this work, it has been demonstrated that it is possible to form NLCs using rhamnolipids as the surfactant. The optimisation of the formulations was based on a Face-Centered Central Composite design being the "optimum" formulation obtained to the ratio of aqueous:lipid phases (AP) $=99 \%$, ratio solid:liquid lipids $(S)=20 \%$ and concentration of rhamnolipids (Sf) $=0.05 \%$. With this in mind, the AP and Sf were kept constant at $99 \%$ and $0.05 \%$, respectively, and the influence of S was studied, testing also the percentages of $15 \%$ and $10 \%$. Although lower S results result in larger sizes, it was observed that such formulations present better stability at 4,25 and $37{ }^{\circ} \mathrm{C}$. So that the best formulation was the NLC 3 with the following composition: AP $=99 \%, \mathrm{~S}=10 \%$ and $\mathrm{Sf}=0.05 \%$. This formulation was more stable at $4{ }^{\circ} \mathrm{C}$ being the only one that continues stable after 30 days. Using DSC and X-ray was possible to confirm that occurs an association between the GM and the MCT and structures with less ordered crystals are formed. The results obtained showed the possibility of controlling stability and crystallinity of rhamnolipids-based NLCs, that can be explored in further developments for the encapsulation and release of bioactive compounds.

\section{CRediT authorship contribution statement}

Maria A. Azevedo: Conceptualization, Investigation, Writing original draft. Miguel A. Cerqueira: Conceptualization, Writing - review \& editing, Supervision. Pablo Fuciños: Methodology, Writing review \& editing. Bruno F.B. Silva: Methodology, Writing - review \& editing. José A. Teixeira: Supervision. Lorenzo Pastrana: Supervision.

\section{Declaration of Competing Interest}

The authors declare that they have no known competing financial interests or personal relationships that could have appeared to influence the work reported in this paper.

\section{Acknowledgements}

Maria A. Azevedo (SFRH/BD/123364/2016) is the recipient of a fellowship from Fundação para a Ciência e Tecnologia (FCT, Portugal). This study was supported by FCT under the scope of the strategic funding of UID/BIO/04469/2013 unit and COMPETE 2020 (POCI-010145-FEDER-006684) and BioTecNorte operation (NORTE-01-0145FEDER-000004) funded by the European Regional Development Fund under the scope of Norte2020 - Programa Operacional Regional do Norte. The authors would like to thank the H2020 MSCA-RISE project FODIAC_Food for Diabetes and Cognition (reference number 778388) and the Comissão de Coordenação e Desenvolvimento Regional do Norte (CCDR-N) project "Nanotechnology based functional solutions" (No. NORTE01- 0145-FEDER-000019). The authors would like to acknowledge to Oliver Schraidt from INL for his kind assistance using TEM.

\section{Appendix A. Supplementary data}

Supplementary data to this article can be found online at https://doi. org/10.1016/j.foodchem.2020.128670.

\section{References}

Azevedo, M. A., Bourbon, A. I., Vicente, A. A., \& Cerqueira, M. A. (2014). Alginate/ chitosan nanoparticles for encapsulation and controlled release of vitamin B2.
International Journal of Biological Macromolecules, 71, 141-146. https://doi.org/ 10.1016/j.ijbiomac.2014.05.036.

Babazadeh, A., Ghanbarzadeh, B., \& Hamishehkar, H. (2017). Formulation of food grade nanostructured lipid carrier (NLC) for potential applications in medicinal-functional foods. Journal of Drug Delivery Science and Technology, 39, 50-58. https://doi.org/ 10.1016/j.jddst.2017.03.001.

Bai, L., \& McClements, D. J. (2016). Formation and stabilization of nanoemulsions using biosurfactants: Rhamnolipids. Journal of Colloid and Interface Science, 479, 71-79. https://doi.org/10.1016/j.jcis.2016.06.047.

Banat, I. M., Franzetti, A., Gandolfi, I., Bestetti, G., Martinotti, M. G., Fracchia, L., Smyth, T. J., \& Marchant, R. (2010). Microbial biosurfactants production, applications and future potential. Applied Microbiology and Biotechnology, 87(2), 427-444. https://doi.org/10.1007/s00253-010-2589-0.

Beloqui, A., del Pozo-Rodríguez, A., Isla, A., Rodríguez-Gascón, A., \& Solinís, M.Á. (2017). Nanostructured lipid carriers as oral delivery systems for poorly soluble drugs. Journal of Drug Delivery Science and Technology, 42, 144-154. https://doi.org/ 10.1016/j.jddst.2017.06.013.

Bouxsein, N. F., Leal, C., McAllister, C. S., Ewert, K. K., Li, Y., Samuel, C. E., \& Safinya, C. R. (2011). Two-Dimensional Packing of Short DNA with Nonpairing Overhangs in Cationic Liposome-DNA Complexes: From Onsager Nematics to Columnar Nematics with Finite-Length Columns. Journal of the American Chemical Society, 133(19), 7585-7595. https://doi.org/10.1021/ja202082c.

Box, G., Hunter, J. S., \& G.Hunter, W. (2005). Statistics for Experimenters: Design, Innovation and Discovery (2nd ed.). Hoboken, New Jersey: John Wiley \& Sons, Inc. https://doi.org/10.1198/tech.2006.s379.

Castelli, F., Puglia, C., Sarpietro, M. G., Rizza, L., \& Bonina, F. (2005). Characterization of indomethacin-loaded lipid nanoparticles by differential scanning calorimetry. International Journal of Pharmaceutics, 304(1-2), 231-238. https://doi.org/10.1016/ j.ijpharm.2005.08.011.

Cerqueira, M. A., Pinheiro, A. C., Silva, H. D., Ramos, P. E., Azevedo, M. A., FloresLópez, M. L., Rivera, M. C., Bourbon, A. I., Ramos, O. L., \& Vicente, A. A. (2014). Design of Bio-nanosystems for Oral Delivery of Functional Compounds. Food Engineering Reviews, 6(1-2), 1-19. https://doi.org/10.1007/s12393-013-9074-3.

Dai, L., Li, R., Wei, Y., Sun, C., Mao, L., \& Gao, Y. (2018). Fabrication of zein and rhamnolipid complex nanoparticles to enhance the stability and in vitro release of curcumin. Food Hydrocolloids, 77, 617-628. https://doi.org/10.1016/j. foodhyd.2017.11.003.

Dai, L., Zhou, H., Wei, Y., Gao, Y., \& McClements, D. J. (2019). Curcumin encapsulation in zein-rhamnolipid composite nanoparticles using a pH-driven method. Food Hydrocolloids, 93(February), 342-350. https://doi.org/10.1016/j. foodhyd.2019.02.041.

Egelhaaf, S., Olsson, U., Schurtenberger, P., Morris, J., \& Wennerström, H. (1999). Quantitative measurements of Ostwald ripening using time-resolved small-angle neutron scattering. Physical Review E, 60(5), 5681-5684. https://doi.org/10.1103/ PhysRevE.60.5681.

Fasolin, L. H., Cerqueira, M. A., Pastrana, L. M., Vicente, A. A., \& Cunha, R. L. (2018). Thermodynamic, rheological and structural properties of edible oils structured with LMOGs: Influence of gelator and oil phase. Food Structure, 16(January), 50-58. https://doi.org/10.1016/j.foostr.2018.03.003.

Gudiña, E. J., Rodrigues, A. I., Alves, E., Domingues, M. R., Teixeira, J. A., \& Rodrigues, L. R. (2015). Bioconversion of agro-industrial by-products in rhamnolipids toward applications in enhanced oil recovery and bioremediation. Bioresource Technology, 177, 87-93. https://doi.org/10.1016/j. biortech.2014.11.069.

Henkel, M., Geissler, M., Weggenmann, F., \& Hausmann, R. (2017). Production of microbial biosurfactants : Status quo of rhamnolipid and surfactin towards largescale production. Biotechnology Journal, 12, 1-10. https://doi.org/10.1002/ biot. 201600561.

Himawan, C., Starov, V. M., \& Stapley, A. G. F. (2006). Thermodynamic and kinetic aspects of fat crystallization. Advances in Colloid and Interface Science, 122(1-3), 3-33. https://doi.org/10.1016/j.cis.2006.06.016.

Hu, F.-Q. Q., Jiang, S.-P. P., Du, Y.-Z. Z., Yuan, H., Ye, Y.-Q. Q., \& Zeng, S. (2006). Preparation and characteristics of monostearin nanostructured lipid carriers. International Journal of Pharmaceutics, 314(1), 83-89. https://doi.org/10.1016/j ijpharm.2006.01.040.

Jenning, V., Thünemann, A. F., \& Gohla, S. H. (2000). Characterisation of a novel solid lipid nanoparticle carrier system based on binary mixtures of liquid and solid lipids. International Journal of Pharmaceutics, 199(2), 167-177. https://doi.org/10.1016/ S0378-5173(00)00378-1.

Kanwar, R., Gradzielski, M., Prevost, S., Appavou, M.-S., \& Mehta, S. K. (2019). Experimental validation of biocompatible nanostructured lipid carriers of sophorolipid: Optimization, characterization and in-vitro evaluation. Colloids and Surfaces B: Biointerfaces, 181, 845-855. https://doi.org/10.1016/j. colsurfb.2019.06.036.

Kuan, C.-Y., Yee-Fung, W., Yuen, K.-H., \& Liong, M.-T. (2012). Nanotech: Propensity in foods and bioactives. Critical Reviews in Food Science and Nutrition, 52(1), 55-71. https://doi.org/10.1080/10408398.2010.494259.

Marques, A. M., Azevedo, M. A., Teixeira, J. A., Pastrana, L. M., Gonçalves, C., \& Cerqueira, M. A. (2019). Engineered Nanostructures for Enrichment and Fortification of Foods. In G. Molina, Inamuddin, F. M. Pelissari, \& A. M. Asiri (Eds.), Food Applications of Nanotechnology (First, pp. 60-96). Boca Raton (FL): CRC Press - Taylor \& Francis Group. https://doi.org/10.1201/9780429297038.

Müller, F., Hönzke, S., Luthardt, W.-O., Wong, E. L., Unbehauen, M., Bauer, J., Rademann, J. (2017). Rhamnolipids form drug-loaded nanoparticles for dermal drug delivery. European Journal of Pharmaceutics and Biopharmaceutics, 116, 31-37. https://doi.org/10.1016/j.ejpb.2016.12.013. 
Nahr, F. K., Ghanbarzadeh, B., Hamishehkar, H., \& Kafil, H. S. (2018). Food grade nanostructured lipid carrier for cardamom essential oil: Preparation, characterization and antimicrobial activity. Journal of Functional Foods, 40, 1-8. https://doi.org/10.1016/j.jff.2017.09.028.

Rodrigues, L. R. (2015). Microbial surfactants: Fundamentals and applicability in the formulation of nano-sized drug delivery vectors. Journal of Colloid and Interface Science, 449, 304-316. https://doi.org/10.1016/j.jcis.2015.01.022.

Salminen, H., Helgason, T., Kristinsson, B., Kristbergsson, K., \& Weiss, J. (2013). Formation of solid shell nanoparticles with liquid $\omega-3$ fatty acid core. Food Chemistry, 141(3), 2934-2943. https://doi.org/10.1016/j.foodchem.2013.05.120.

Santos, V. da S., Braz, B. B., Silva, A. Á., Cardoso, L. P., Ribeiro, A. P. B., \& Santana, M. H. A. (2019). Nanostructured lipid carriers loaded with free phytosterols for food applications. Food Chemistry, 298(May), 125053. https://doi.org/10.1016/j. foodchem.2019.125053.

Silva, B. F. B., Majzoub, R. N., Chan, C.-L., Li, Y., Olsson, U., \& Safinya, C. R. (2014). PEGylated cationic liposome-DNA complexation in brine is pathway-dependent. Biochimica et Biophysica Acta (BBA) - Biomembranes, 1838(1), 398-412. https://doi. org/10.1016/j.bbamem.2013.09.008.

Soleimanian, Y., Goli, S. A. H., Varshosaz, J., \& Sahafi, S. M. (2018). Formulation and characterization of novel nanostructured lipid carriers made from beeswax, propolis wax and pomegranate seed oil. Food Chemistry, 244, 83-92. https://doi.org/ 10.1016/j.foodchem.2017.10.010.

Tamjidi, F., Shahedi, M., Varshosaz, J., \& Nasirpour, A. (2013). Nanostructured lipid carriers (NLC): A potential delivery system for bioactive food molecules. Innovative Food Science \& Emerging Technologies, 19, 29-43. https://doi.org/10.1016/j. ifset.2013.03.002.

Teeranachaideekul, V., Muller, R. H., \& Junyaprasert, V. B. (2007). Encapsulation of ascorbyl palmitate in nanostructured lipid carriers (NLC)—Effects of formulation parameters on physicochemical stability. International Journal of Pharmaceutics, 340 (1-2), 198-206. https://doi.org/10.1016/j.ijpharm.2007.03.022.

Uluata, S., McClements, D. J., \& Decker, E. A. (2016). Riboflavin-induced oxidation in fish oil-in-water emulsions: Impact of particle size and optical transparency. Food Chemistry, 213, 457-461. https://doi.org/10.1016/j.foodchem.2016.06.103.

Varshosaz, J., Eskandari, S., \& Tabbakhian, M. (2012). Freeze-drying of nanostructure lipid carriers by different carbohydrate polymers used as cryoprotectants. Carbohydrate Polymers, 88(4), 1157-1163. https://doi.org/10.1016/j. carbpol.2012.01.051.

Wang, F. C., \& Marangoni, A. G. (2014). Nature and dynamics of monostearin phase transitions in water: Stability and the sub- $\alpha$-gel phase. RSC Advances, 4(92), 50417-50425. https://doi.org/10.1039/C4RA08648A.

Wei, Y., Yu, Z., Lin, K., Sun, C., Dai, L., Yang, S., Mao, L., Yuan, F., \& Gao, Y. (2019). Fabrication and characterization of resveratrol loaded zein-propylene glycol alginate-rhamnolipid composite nanoparticles: Physicochemical stability, formation mechanism and in vitro digestion. Food Hydrocolloids, 95, 336-348. https://doi.org/ 10.1016/j.foodhyd.2019.04.048.

Weiss, J., Decker, E. A., McClements, D. J., Kristbergsson, K., Helgason, T., \& Awad, T. (2008). Solid Lipid Nanoparticles as Delivery Systems for Bioactive Food Components. Food Biophysics, 3(2), 146-154. https://doi.org/10.1007/s11483-0089065-8.

Yi, G., Son, J., Yoo, J., Park, C., \& Koo, H. (2019). Rhamnolipid nanoparticles for in vivo drug delivery and photodynamic therapy. Nanomedicine: Nanotechnology. Biology and Medicine, 19, 12-21. https://doi.org/10.1016/j.nano.2019.03.015.

Zheng, M., Falkeborg, M., Zheng, Y., Yang, T., \& Xu, X. (2013). Formulation and characterization of nanostructured lipid carriers containing a mixed lipids core. Colloids and Surfaces A: Physicochemical and Engineering Aspects, 430, 76-84. https:// doi.org/10.1016/j.colsurfa.2013.03.070. 